\title{
Las rutas del vino del Empordà y del Priorat y su dinámica relacional. Dos destinos con diferente grado de desarrollo del turismo enológico y una dinámica relacional similar
}

\section{The routes of the wine of the Empordà and the Priorat and their relational dynamics. Two destinations with different degree of development of the enologic tourism and similar relational dynamics}

\author{
Josep $\mathrm{M}^{\mathrm{a}}$ Prat Forga y Gemma Cànoves Valiente ${ }^{1}$
}

\section{INTRODUCCIÓN}

En la actualidad, en un mundo globalizado, los turistas, cada vez más experimentados, presentan nuevas exigencias y motivaciones, reclamando la máxima calidad posible, un respeto medioambiental y un trato cada vez más personalizado (Bigné et al., 2000). Ante esta situación, los destinos turísticos, unos para evitar su estancamiento y declive -dentro del ciclo de vida de Butler (1980)-, y otros para impulsar su desarrollo, están reinventando permanentemente su oferta de productos y servicios turísticos, siendo la búsqueda de nuevos productos una de las estrategias más comunes (Bigné et al., 2000; Ritchie y Zins, 1978).

En este artículo se presenta una de estas iniciativas, el turismo enológico y las rutas del vino, como un recurso de gran potencial turístico y cultural,

${ }^{1}$ Universitat Autònoma de Barcelona. Departamento de Geografía. josepmaria.prat@uab. cat; gemma.canoves@uab.cat. Proyecto RETIS CSO2013-41374-R . 
tanto desde el punto de vista de la oferta como de la demanda (Elías, 2006; Mondéjar y Sevilla, 2008). Así, los agentes locales han promovido nuevos productos turísticos y nuevas actividades, como actividad complemento en el caso de los destinos maduros -cuya motivación principal es otra- o como medio para desarrollar un turismo específico y con una señal de identidad propia -el turismo enológico-, que, apoyándose en el consumo de unos recursos tradicionales, ayude al desarrollo económico de unos territorios en declive, generando empleo y consumiendo los productos locales (López- Guzmán y Sánchez, 2008). En ambos casos, se trata de una oferta basada en degustaciones («catas») que se realizan mediante visitas planificadas a bodegas donde, además, pueden realizarse otras actividades, incluyendo la degustación de otros productos gastronómicos típicos, generalmente elaborados artesanalmente, los cursillos de catas de vinos y las visitas a los viñedos.

Desde el punto de vista de la demanda, con las rutas del vino se consume un turismo cultural-enológico, que presenta una gran capacidad de atracción para un público ávido de nuevas experiencias, más personalizadas, por lo que se convierte en un recurso enormemente valorado por parte de un determinado segmento de la población (Middleton, 1994; Castillo, 2007).

Ante esta situación, en este trabajo se analizan y comparan dos rutas del vino desarrolladas en dos destinos con diferentes estructuras turísticas, a través de la dinámica relacional generada a través de Internet por los agentes implicados con este turismo enológico. El objetivo es comprobar si existe una relación directa entre esta dinámica relacional y el grado de desarrollo de dicho turismo, de modo que la presencia en Internet de una red social más densa implique un mayor desarrollo del citado turismo, o bien si en dicho desarrollo también influye la estructura turística presente actualmente en el destino.

Así pues, la hipótesis que aquí se pretende demostrar es comprobar si dos destinos con diferente estructura turística presentan una dinámica relacional parecida en la red formada por los agentes involucrados con un determinado producto turístico -las rutas del vino-, cuyo grado de desarrollo es similar en ambos territorios.

Por ello, en este artículo, que forma parte de un proyecto de mayor alcance sobre el turismo de proximidad, después de presentar las principales características del turismo enológico y las rutas del vino, y explicar la metodología aquí utilizada, se ha realizado el análisis de las redes sociales (ARS) y de sus contenidos (ACRS), para dos rutas del vino localizadas en dos territorios cuya estructura turística es muy distinta. Por una parte, el Empordà (incluyendo las comarcas del Alt y Baix Empordà) y, por la otra, el Priorat. El primero, situado en una zona con un turismo de litoral muy consolidado y maduro -con el re- 
clamo de la Costa Brava-, que busca nuevos productos que complementen su oferta turística, de manera que, además de atraer nuevos turistas, una parte de los habituales puedan satisfacer sus motivaciones culturales-enológicas, mediante las visitas a las bodegas y la cata de sus vinos. El segundo, el Priorat, una comarca en declive demográfico y económico, cuyo desarrollo turístico es muy reducido pero que se aferra a este nuevo producto como herramienta para su reactivación, ya que con estas experiencias, los turistas tienen la oportunidad de conocer y saborear los vinos más prestigiosos del territorio y, a la vez, conocer su paisaje, su cultura, sus recursos y su gente (Middleton, 1994).

Así, se han comparado las relaciones sociales establecidas en ambas redes a través de Internet y se ha confirmado la hipótesis planteada previamente, ya que se ha llegado a la conclusión de que, aunque el grado de desarrollo y la estructura turística de ambos destinos sea muy diferente, si la dinámica relacional generada por las redes de los agentes involucrados directamente en el desarrollo de un determinado producto turístico -como son las rutas del vino- es similar, entonces el grado de desarrollo de este tipo de turismo es parecido en ambos territorios; lo que puede servir como pauta para futuras estrategias de desarrollo de este turismo -en nuestro caso el turismo enológicoen diferentes tipos de destinos.

\section{EL TURISMO ENOLÓGICO Y LAS RUTAS TURÍSTICAS}

Tal como se ha indicado anteriormente, la industria del turismo ha evolucionado rápidamente en estos últimos años, creando nuevos productos, y una de sus apuestas ha sido el turismo enológico (Elías, 2006), del que Wolf (2002) ya indicó que servía para buscar, saborear y disfrutar de la bebida como una experiencia única y memorable, que puede realizarse a lo largo de todo el año (ICTA, 2012). De esta manera, se relacionan cultura y bebida (Kwangmin y Jinhoo, 2010), siendo considerada la enología como base de la cultura culinaria, ya que lo que la gente come y bebe suele estar asociado a los territorios, que distinguen y demarcan estas fronteras culturales (Hjalager y Richards, 2002).

Por ello, las percepciones sensoriales juegan un importante papel psicológico y fisiológico en las apreciaciones de estos turistas (Boniface, 2003; Long, 2004), y forman parte del conjunto de atributos que modelan las atracciones turísticas de los destinos (Warde, 2002), sirviendo, además, como reclamo promocional para futuras repeticiones o para atraer nuevos clientes, por lo que se convierten en un interesante instrumento de mercadotecnia (Richards, 2002). 
Con este consumo turístico se refuerza la propia identidad de estos turistas enológico-culturales, ya que se trata de unas prácticas que suelen estar asociadas a unas determinadas clases sociales (Hashimoto y Telfer, 2006; Smith y Xiao, 2008; Mkono, 2011), y pudiendo llegar a modificarles sus gustos, hábitos y preferencias (Johns y Clarke, 2001; Castillo, 2007). En este contexto, el turismo enológico se ha asentado como un nuevo nicho de mercado que ha experimentado un importante auge, de modo que hay gente que viaja a un determinado destino expresamente para saborear sus vinos como parte de su formación cultural personal (Chulwon y Taesuk, 2005; Kivela y Crotts, 2006; López-Guzmán y Sánchez, 2008).

En general, el perfil de este tipo de turistas es de un nivel socio-cultural y económico alto y medio-alto, que presenta unas exigencias superiores a la media de los turistas y que suele poner la calidad del producto consumido por encima de otras consideraciones, tales como el precio (Long, 2004). Se trata de un turista exigente y crítico, conocedor de una oferta que no solamente le gusta sino que forma parte de sus aficiones, consumiendo esta experiencia como una vivencia única y una inversión en cultura. Por ello suele informarse sobre el tema a través de artículos en publicaciones, especializadas o no, y tiene muy presente las recomendaciones dadas tanto por los expertos como por sus amigos y conocidos (Middleton, 1994).

También es cierto que se trata de un turismo donde, además de la calidad, la estética, la organización y la prestación del servicio conllevan un atractivo necesario no sólo para atraer al turista sino para que, con la satisfacción por el cumplimiento de sus expectativas, éste pueda ser fidelizado, repita las visitas y se convierta en prescriptor del producto turístico-enológico (Long, 2004).

Además, este tipo de turismo también juega un papel importante en el desarrollo económico - generando nuevos puestos de trabajo y vendiendo más productos del territorio- (Alonso y Méndez, 2000; Alonso, 2003) y en la afirmación de la cultura y de la identidad locales, por lo que, en muchas ocasiones, estos son los únicos factores que tienen en común los turistas y los lugareños (Acercosa, 2006; Martínez Puche y Jover, 2012).

Así, según se desprende del estudio sobre la oferta enoturística de las rutas del vino, realizado en el segundo semestre del año 2009 por el Observatorio Turístico de las Rutas del Vino de España sobre 532 cuestionarios recibidos de más del 55\% de las empresas asociadas (bodegas, alojamientos, restaurantes, museos y centros de interpretación, enotecas y comercios, agencias receptivas, oficias de turismo y otros servicios) a las trece rutas entonces certificadas (Bullas, La Mancha, Jerez, Jumilla, Montilla-Moriles, Navarra, Penedès, Rías Baixas, Rioja Alavesa, Somontano, Tacoronte-Acentejo, Utiel-Requena, Ribeiro) y 
una más aún no certificada (Ribera del Duero), el 52,8\% de las respuestas recibidas consideraban que la adhesión a la Ruta del Vino había sido positiva para su empresa.

Dicha repercusión se focaliza principalmente en un aumento del volumen de clientes (en el caso de más del 51\% de los encuestados), una mayor rentabilidad del negocio (casi el 49\% de los encuestados así lo afirmaron) y la creación de nuevos puestos de trabajo (el 44\% de las empresas contestaron que habían creado al menos medio puesto de trabajo más; destacando que en el $14 \%$ de los casos se crearon entre dos y cinco, y en un $4 \%$ más de cinco nuevos puestos).

Paralelamente, este mayor desarrollo del turismo del vino ha exigido una adaptación y mejora de dichas empresas, con el objetivo de ser más competitivas ante esta nueva demanda, lo que ha provocado la necesidad de realizar algunas inversiones en el acondicionamiento, la señalización y el material promocional, de modo que cerca del $57 \%$ de los encuestados afirmaron haber realizado dicha inversión.

También destacan las nuevas actividades que las bodegas tradicionales han incorporado a su oferta, como las visitas a los viñedos, degustaciones, exposiciones, jornadas gastronómicas, celebración de eventos y la posibilidad de disponer de alojamiento en la propia bodega. Todo ello ha servido para que estos establecimientos manifiesten un alto nivel de satisfacción con su pertenencia a las Rutas. Así, más del 78\% de las 222 que respondieron al cuestionario indicaron esta circunstancia (Observatorio del Turismo, 2010). Hay que tener en cuenta que el turismo del vino aporta a las bodegas numerosos beneficios vinculados a la generación de marca y fidelización de clientes, pero también obtienen ingresos directos por la venta de vino y otros productos y servicios (Mondéjar y Sevilla, 2008).

En cuanto a las oficinas de turismo, a pesar de que Internet cada vez es más utilizado para informarse sobre los destinos y sus ofertas, aquellas todavía tienen un importante papel en el aprovechamiento y en el disfrute de la experiencia turística, especialmente durante su estancia en el destino (Martínez Puche y Jover, 2012). Así, la vinculación de estas oficinas con los restantes agentes involucrados con las Rutas es muy estrecha, principalmente en la distribución de material promocional e información sobre las visitas guiadas (Castillo, 2007).

Por todo ello, en estos últimos años se han incrementado tanto las visitas individuales de turismo enológico como las rutas planificadas, como medio para estimular el desarrollo local y satisfacer las demandas culturales de este tipo de turistas (Elías, 2006; Castillo, 2007; Zillinger, 2007; Mondéjar y Sevi- 
lla, 2008). Así, por ejemplo, no hay que olvidar que hay más visitantes en las bodegas de las rutas del vino del Valle de Napa, en California (USA), que en el Disneyworld californiano (Vogeler y Hernández, 2000).

\section{LAS RUtAS DEL VINo EN El EMPORdÀ y EN El PRIORAT}

Seguidamente se describen las principales característica de los dos territorios seleccionados: las dos comarcas ampurdanesas y el Priorato, cuya localización se puede observar en el mapa siguiente (figura 1) y cuyos principales

FIGURA 1

SITUACIÓN DE LAS COMARCAS ANALIZADAS

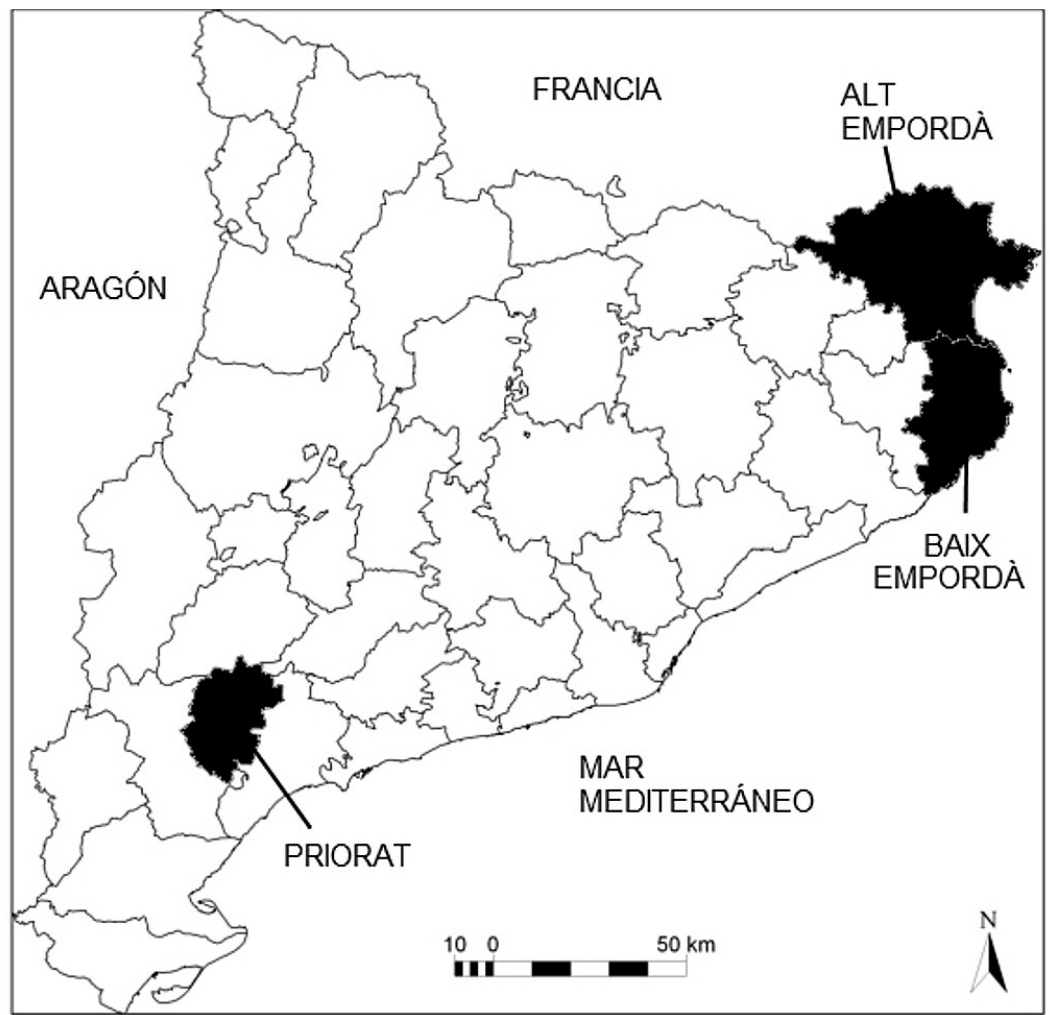

Fuente: elaboración propia.

Estudios Geográficos, Vol. LXXV, 277, pp. 683-705, julio-diciembre 2014

ISSN: 0014-1496, eISSN: 1988-8546, doi: 10.3989/estgeogr.201422 
CuAdro 1

RESUMEN ESTADÍSTICO DE LAS COMARCAS SELECCIONADAS

\begin{tabular}{lccccc}
\hline & Año & $\begin{array}{c}\text { Alt } \\
\text { Empordà }\end{array}$ & $\begin{array}{c}\text { Baix } \\
\text { Empordà }\end{array}$ & $\begin{array}{c}\text { Total } \\
\text { Empordà }\end{array}$ & Priorat \\
\hline Población (hab.) & 2011 & 140.426 & 133.116 & 273.542 & 10.087 \\
Superficie (km2) & & $1.357,5$ & 701,7 & $2.059,2$ & 498,6 \\
Densidad (hab./km2) & 2011 & 103,4 & 189,7 & 132,8 & 20,2 \\
Segundas residencias & 2001 & 46.041 & 45.269 & 91.310 & 2.510 \\
Plazas hoteleras & 2011 & 14.537 & 13.815 & 28.352 & 288 \\
Plazas en campings & 2011 & 38.385 & 61.116 & 99.501 & 1.023 \\
Plazas de turismo rural & 2011 & 1.184 & 542 & 1.726 & 354 \\
Restaurantes & 2005 & 727 & 690 & 1.417 & 32 \\
\hline
\end{tabular}

Fuente: Elaboración propia a partir de Idescat (2012).

datos sociodemográficos y de desarrollo turístico se presentan en la tabla siguiente (cuadro 1), teniendo en cuenta que el desarrollo del viñedo está muy ligado a las condiciones del medio natural, especialmente el clima, el tipo suelo y el relieve, los cuales influyen decisivamente en el desarrollo vegetativo de estas plantas (Fernández Portela, 2012).

\section{a) El caso del Empordà}

El Empordà es una comarca natural e histórica situada en el noreste de la provincia de Girona, que está comprendida entre las sierras de la Albera, al norte; las Gavarres, al suroeste; y el mar Mediterráneo, al este y al sur. Limita al norte con las comarcas históricas del Vallespir y el Rosellón, actualmente dentro del departamento de los Pirineos Orientales, en la región francesa del Languedoc-Rosellón, y al oeste con las comarcas de la Garrotxa, el Pla de l'Estany, el Gironés y la Selva. Sin embargo, en 1936 quedó dividido administrativamente en dos comarcas: el Alt Empordà -con capital en Figueres- y el Baix Empordà -con capital en La Bisbal-. 
Su relieve se distingue por la llanura -donde predominan los suelos de origen aluvial-y las montañas -donde los suelos son de pizarra y graníticos-. Su clima es templado y mediterráneo, con inviernos suaves -con pocas heladasy veranos calurosos, atemperados por las brisas marinas. Como rasgo característico, el viento dominante es la Tramontana, con rachas que a menudo superan los 120 kilómetros por hora, y cuyos efectos son muy beneficiosos para el buen estado sanitario de las viñas (Fernández Portela, 2012).

En su litoral se encuentra parcialmente la Costa Brava, lo que sitúa este territorio, con una densidad media de población residente de $132,8 \mathrm{hab} / \mathrm{km}^{2}$, en un lugar preeminente dentro del sector turístico catalán y español, con un PIB per cápita alrededor de los 24.000 euros, y con una oferta de 28.352 plazas hoteleras, 1.726 plazas de turismo rural, 99,501 plazas de camping y 91.310 segundas residencias (fuente: Idescat, 2012), tal como se detalla en la tabla anterior (cuadro 1).

Por otra parte, presenta una importante oferta enológica, basada en los vinos de la Denominación de Origen (DO) Empordà -ocupando una superficie de 2.070 ha y produciendo más de $60.000 \mathrm{Hl}$. anuales (Incavi, 2012)-, con una zona de producción que se extiende desde las faldas de las sierras de Rodes y de las Alberas, en la frontera francesa -en un arco que va desde el cabo de Creus a la Garrotxa d'Empordà-, hasta la sierra de las Gavarras, al sur. De esta manera, la DO ocupa una gran heterogeneidad de terrenos llanos y en pendiente -desde el nivel del mar hasta un máximo de $260 \mathrm{~m}$ de altitud-, que mayoritariamente son de textura arenosa, ácidos y pobres en materia orgánica (Fernández Portela, 2012).

Con el fin de consolidar esta industria vitivinícola y, a su vez, desarrollar el turismo enológico en el territorio, una de las principales iniciativas ha sido la creación de una ruta del vino, impulsada y coordinada por el Patronato de Turismo de Girona, con el apoyo económico de la Secretaría General de Turismo de España-que promueve el turismo enológico en aquellas zonas de España vinculadas a la viticultura, con el objetivo de contribuir a su desarrollo económico y social-, la participación de las principales bodegas de la zona y la colaboración de las oficinas de turismo, las instituciones públicas y las asociaciones empresariales privadas; todos los cuales, a pesar de tener diferentes objetivos propios, presentan un idéntico interés: la promoción de los vinos del Empordà y el desarrollo de un turismo que permita compaginar el interés por la enología con la atracción histórica, cultural, arquitectónica y paisajística de estos territorios.

Se trata de una ruta que ha sido incluida en las Rutas del Vino de la Asociación Española de las Ciudades del Vino y que ha editado la Guía de Enoturismo 
del Empordà, que da detalles sobre las bodegas que pueden ser visitadas en estas dos comarcas ampurdanesas, así como la relación de 54 hoteles y restaurantes, recomendados por su cuidado al vino local. Además, se proponen una serie de actividades culturales complementarias, como las visitas a los museos del vino de la provincia, a las fábricas de tapones de corcho y a los monasterios -antiguos depósitos arqueológicos- para conocer la historia y la evolución de este producto.

\section{b) El caso del Priorat}

El Priorat es una comarca situada al sur de Catalunya, en la provincia de Tarragona, que limita con las comarcas del Baix Camp, la Ribera d'Ebre, las Garrigues y la Conca de Barberà. Como que situada entre la sierra de Montsant -que la comprende-, al norte; y el conjunto formado por la Mola de Colldejou, la sierra de Llavería y la sierra de Santa Marina, al sur; presenta una orografía muy accidentada y difícil para el cultivo de la vid, con terrenos de poca extensión y pequeñas propiedades -lo cual no permite grandes rendimientos-, proliferando buen número de pequeñas bodegas.

$\mathrm{Su}$ clima es continental, seco y caluroso en verano y frío en invierno. En cuanto a su relieve, en su sector central proliferan las rocas del carbonífero (principalmente pizarras), con un grado de metamorfismo variable. En estas condiciones, por su situación geográfica, su clima, su relieve y su falta de comunicaciones, la comarca ha perdido mucha población durante el siglo xx, presentando una baja densidad $\left(20,2 \mathrm{hab} / \mathrm{km}^{2}\right)$, solamente 2.510 segundas residencias y una muy limitada oferta turística (288 plazas hoteleras, 1.023 plazas de campings, 354 de turismo rural y 32 restaurantes), lo que contrasta grandemente con la estructura turística del Empordà, uno de los territorios catalanes con mayor desarrollo del turismo.

Sin embargo, en estos últimos años se percibe una cierta estabilización demográfica, vinculada a la recuperación económica de la comarca, en gran parte gracias a la industria vitivinícola, con una larga tradición en el cultivo de la viña, ya que, desde el siglo xII, la Cartuja de Scala Dei fomentó su cultivo, el cual pasó a ser en el siglo XviII el producto más importante de este territorio.

Con una superficie de cultivo aproximada de 3.400 ha (Incavi, 2012), esta comarca, que presenta todo tipo de suelos, junto con la del relieve (de 100 a $750 \mathrm{~m}$ de altitud) y una climatología muy peculiar, produce diferentes variedades de uvas que dan como fruto una extensa gama de vinos, que dan 
lugar a dos distintas denominaciones de origen: DO Priorat y DO Montsant. La primera, localizada en el sector central de la comarca, empezó a cultivar la viña en el siglo XII, al amparo de la comunidad cartujana allí establecida hasta la mitad del siglo xix, y constituyendo en 1954 su denominación de origen. La segunda, formada por los vinos de los pueblos situados en un anillo alrededor de la DO Priorat, tenía hasta hace poco tiempo una actividad vinícola dominada por las cooperativas surgidas a principios del siglo $\mathrm{xx}$, aunque actualmente podemos encontrar también bodegas privadas de gran calidad.

Ante esta situación, en los últimos años, con el objetivo de consolidar esta nueva pujanza de la industria vitivinícola en la comarca, se están elaborando unos vinos cuya promoción, comercialización, desarrollo y diseño, desde mediados de la década de los setenta, están orientados a obtener unos productos de calidad que puedan competir con éxito en el mercado nacional e internacional. Para mejorar su conocimiento y fomentar el desarrollo del turismo enológico en este territorio, desde la Oficina de Turismo del Priorat se ha estructurado una oferta con 12 bodegas visitables para la DO Montsant y 28 para la DO Priorat.

\section{METOdOLOGÍA, FUENTES DE INFORMACIÓN Y VARIABLES UTILIZADAS}

En los dos casos seleccionados, nos encontramos ante unos territorios con una estructura turística muy diferente, pero con un turismo enológico cuyas rutas del vino presentan similares niveles de desarrollo -superando ambas la cifra de 10.000 visitantes al año y con un crecimiento anual sostenido (Incavi, 2012)-. En su creación y promoción se han implicado los diferentes agentes relacionados con este tipo de turismo -proveedores, instituciones y organismos públicos, asociaciones-, formándose unas redes sociales cuyas relaciones a través de Internet están ayudando a su consolidación (Ejarque, 2005).

Por otra parte, en la literatura académica relativa al turismo enológico, el empleo del concepto de red social, y de su dinámica relacional, todavía no está demasiado desarrollado (McCurley y Linchan, 1996; McBee, 2002), a pesar de que el turismo es un sistema conectado en red, cuyos actores cooperan y compiten en una evolución dinámica (Scott, et al. 2008).

Las técnicas de análisis de redes socales (ARS) proporcionan un medio muy útil para conceptuar, visualizar y analizar estos complejos juegos de relaciones (Scott, et al. 2008), presentándose dos aproximaciones diferentes para 
su estudio. Una de ellas se basa en la búsqueda de la cohesión de la red -que es el método utilizado en este trabajo- y la otra lo hace en la búsqueda de las posiciones estructurales; aunque ambas presentan resultados similares (Haythornthwaite, 1996; Molina, 2001).

En el presente trabajo, los tres diferentes tipos de indicadores seleccionados para medir la cohesión de la red son:

a) Densidad: mide la proporción de relaciones existentes en la red respecto a las posibles (Hanneman, 2000; Merinero y Pulido, 2009)

b) Cohesión: indica el esfuerzo necesario para que un actor alcance a otro en la red, señalando cual es el grado de compactación de la red («compactness») (Hanneman, 2000; Requena, 2003)

c) Centralidad: indica las relaciones directas que tiene cada actor en la red «negree»); el grado de intermediación de la red («betweenness») y los actores que se encuentran en una mejor posición de intermediarios, lo que les permite controlar los flujos de comunicación; y la cercanía «closeness», que calcula la suma de los caminos más cortos que unen a cada actor con el resto (Hanemann, 2000; Molina, 2001)

Para analizar estos indicadores, y conocer la dinámica relacional de ambas redes, se han analizado las relaciones presentes en Internet entre los diferentes agentes involucrados con las rutas del vino -oficinas y patronatos de turismo, instituciones públicas, asociaciones y bodegas-; con el apoyo de las revistas y folletos publicitarios.

Para ello se ha utilizado el método de la «bola de nieve» («snowballing»), de manera que a partir de un reducido número de actores iniciales (obtenidos desde el Patronato de Turismo de Girona y de Priorat Turisme, respectivamente), con las revisiones de las webs y de los medios impresos consultados, se han ido incorporando nuevos agentes y sus relaciones (Granovetter, 1976; Frank y Snidjers, 1994; Molina, 2001). También se ha obtenido información estadística complementaria a través del Idescat y del Incavi.

Una vez conocidos los agentes involucrados con las redes para el desarrollo de ambas rutas, y clasificados en cuatro grupos: A (organizaciones de turismo); B (instituciones públicas); C (asociaciones y entidades privadas); y D (productores), se han analizado las relaciones presentes entre ellos, mediante el programa Ucinet 6 (Borgatti, et al. 2002), creándose dos matrices adyacentes, binarias, cuadradas y de modo 1 (los mismos actores en las filas y en las columnas): una para la red social creada con la ruta del vino del Empordà (ruta 1) y otra para la red de la ruta del vino del Priorat (ruta 2). 
Después, con el mismo programa Ucinet 6 se ha realizado el correspondiente análisis ARS para cada una de ambas redes, y luego, con NetDraw se ha elaborado su representación gráfica mediante los correspondientes sociogramas.

Finalmente, mediante una serie de entrevistas personales efectuadas entre marzo y septiembre de 2012 a una muestra seleccionada al azar de los agentes obtenidos (en concreto, en las comarcas ampurdanesas se han realizado 30 entrevistas, de las que seis han sido al personal responsable de las oficinas de turismo, seis al de los ayuntamientos, tres al de las asociaciones y quince al de las bodegas; y en el Priorat se han realizado 27 entrevistas, de las que tres ha sido al personal responsable de las oficinas de turismo, siete al de los ayuntamientos, tres al de las asociaciones y catorce al de las bodegas) se han confirmado las relaciones encontradas a través de Internet y de los medios impresos, y se ha analizado el contenido de dichas relaciones sociales (ARCS).

Dicho análisis ACRS se ha basado en las respuestas obtenidas sobre el tipo de relaciones establecidas en la red entre sus miembros (bilaterales o multilaterales), su temporalidad (puntuales o permanentes), el grado de formalización (confianza o contractuales) y su temática (promoción del turismo enológico, información sobre las rutas del vino o desarrollo de la industria vitivinícola).

\section{RESUlTADOS DEL ANÁLISIS REALIZADO}

En el caso de la ruta del Empordà (ruta 1), se obtuvieron 105 agentes distintos, que se agruparon en los cuatro grupos citados anteriormente, tal como se detalla en la tabla siguiente (cuadro 2). El mismo procedimiento se siguió en el caso de la ruta del Priorat (ruta 2), obteniéndose 80 agentes diferentes,

CUADRO 2

GRUPOS DE AGENTES

\begin{tabular}{clcc}
\hline Tipo & \multicolumn{1}{c}{ Descripción } & Ruta 1 & Ruta 2 \\
\hline A & Organismos de turismo (círculos en el sociograma) & 15 & 6 \\
B & Instituciones públicas (cuadrados) & 24 & 14 \\
C & Asociaciones y organizaciones privadas (triángulo) & 29 & 17 \\
D & Productores (triángulo invertido) & 37 & 43 \\
& Total & 105 & 80 \\
\hline
\end{tabular}

Fuente: Elaboración propia. 
que también fueron agrupados en los mismos cuatro grupos. Como se puede observar en la tabla, en ambos casos predominaron los productores de vino, seguidos por las asociaciones y organizaciones privadas.

Una vez creadas las dos matrices adyacentes con los agentes así obtenidos y establecidas sus relaciones con el resto, mediante combinaciones binarias («1» si había relación y «0» si no la había), se han obtenido los resultados del ARS realizado con Ucinet 6 (Borgatti, et al. 2002) para cada una de ambas matrices y el contenido de dichas relaciones -a partir de las entrevistas- y cuyo detalle se presenta en la tabla siguiente (cuadro 3).

\section{CUADRO 3}

ARS Y ACRS

\begin{tabular}{lcc}
\hline & Ruta 1 & Ruta 2 \\
\hline ARS & & \\
\hline Nodos & 105 & 80 \\
\hline DENSIDAD & $17,16 \%$ & $10,16 \%$ \\
Densidad & & \\
\hline COHESIÓN & 1,895 & 1,050 \\
Distancia promedio & 0,575 & 0,900 \\
Grado de compactación («compactness») & & \\
\hline CENTRALIDAD & $21,50 \%$ & $21,32 \%$ \\
Grado de centralización («negree») & $59,78 \%$ & $54,37 \%$ \\
Grado de cercanía («closeness») & $39,07 \%$ & $38,68 \%$ \\
Grado de intermediación («betweenness») & & \\
\hline ACRS & & \\
\hline TIPO DE RELACIONES & $4,57 \%$ & $3,73 \%$ \\
Bilaterales & $95,43 \%$ & $96,27 \%$ \\
Multilaterales & $2,13 \%$ & $5,14 \%$ \\
\hline TEMPORALIDAD DE LAS RELACIONES & $97,87 \%$ & $94,86 \%$ \\
Puntuales & & \\
Permanentes & $78,37 \%$ & $82,75 \%$ \\
\hline FORMALIZACIÓN DE LAS RELACIONES & $21,63 \%$ & $17,25 \%$ \\
Confianza & $54,32 \%$ & $46,24 \%$ \\
Contractuales & $24,12 \%$ & $26,39 \%$ \\
\hline TEMÁTICA DE LAS RELACIONES & $21,56 \%$ & \\
Promoción turismo enológico & & \\
Información sobre las rutas del vino & & \\
Desarrollo industria vitivinícola & & \\
\hline
\end{tabular}

Fuente: elaboración propia con Ucinet 6. 
Como puede comprobarse en la tabla anterior, la red relacional correspondiente a la ruta 1 (ruta del vino del Empordà), presenta una densidad superior a la del Priorat (ruta 2), con un 17\% en el caso de la primera frente al 10\% en el de la segunda. Ello se explica por mayor presencia en el territorio ampurdanés de oficinas de turismo, asociaciones y ayuntamientos. Los correspondientes sociogramas son los siguientes: ruta 1 (figura 2) y ruta 2 (figura 3).

Sin embargo, la cohesión es similar en ambas redes, ya que la ruta 2 , a pesar de ser menor su distancia promedio (1,05 frente al 1,895 de la red de la ruta 1), presenta una compactación mayor (con un índice de compactación de 0,9 frente al 0,575 de la otra red). También la centralidad es parecida en ambas redes, ya que ambas presentan similares valores (alrededor del 21\%). Lo mismo ocurre con la intermediación (alrededor del 39\%) y la cercanía (el 59\% en la ruta 1 y el $54 \%$ de la ruta 2 ).

Por ello, aunque las densidades de las dos redes no son demasiado altas (ambas presentan valores inferiores al 20\%), la dinámica relacional (cohesióncompactación-centralidad-intermediación) de la red de la de la ruta 1 es muy parecida a la de la red de la ruta 2, lo que se corresponde con el similar grado de desarrollo de ambas rutas de turismo enológico en ambos territorios (ambas presentan parecido número de visitantes, según datos facilitados por el Incavi, 2012), a pesar de que en el caso del Empordà, tal como se refleja en el cuadro 1 , nos encontramos ante un destino turístico muy desarrollado y con una estructura consolida.

Con relación a los agentes, los que presentan mayor centralidad, según los resultados del ARS, para la ruta 1 son, por este orden, la Oficina de Turismo Figueres (nodo $n^{\circ} 1$ ), la Oficina de Turismo de Rosas ( $\left.n^{\circ} 2\right)$, la Oficina de Turismo de Portbou ( $\mathrm{n}^{\mathrm{o}} 3$ ), la Oficina de Turismo de Castelló d'Empúries ( $\left.\mathrm{n}^{\circ} 4\right)$ y la Oficina de Turismo de Peralada ( $\left.{ }^{\circ} 5\right)$. A su vez, para la ruta 2, los agentes más centrales son Priorat Turisme (nodo $\mathrm{n}^{\circ} 27$ ), la Asociación de la Ruta de las Bodegas ( $\left.\mathrm{n}^{\circ} 30\right)$, la Oficina de Turismo del Priorat $\left(\mathrm{n}^{\circ} 2\right)$, el Consell Comarcal del Priorat ( $\left.n^{\circ} 10\right)$ y el Ayuntamiento de Falset ( $\left.n^{\circ} 9\right)$.

En la ruta del Empordà (ruta 1) predomina el poder de intermediación de las oficinas de turismo allí situadas, mientras que en el caso de la ruta del Priorat (ruta 2) este papel lo tienen las asociaciones y entidades públicas, que aquí juegan un papel fundamental en el desarrollo del turismo enológico.

Los agentes con mayor grado de centralización en la red de la ruta 1 son todos los anteriores que presentaban mayor poder de intermediación, a los que hay que añadir las oficinas de turismo de Sant Pere Pescador (nodo $n^{\circ} 6$ ) y de Llançà $\left(n^{\circ} 11\right)$, el Consejo Regulador del Vino del Empordà ( $\left.n^{\circ} 33\right)$, la asociación Alt Empordà Turisme ( ${ }^{\circ}$ 49) y el Consorcio del Castell de Peralada 
FIGURA 2

\section{SOCIOGRAMA RUTA 1}

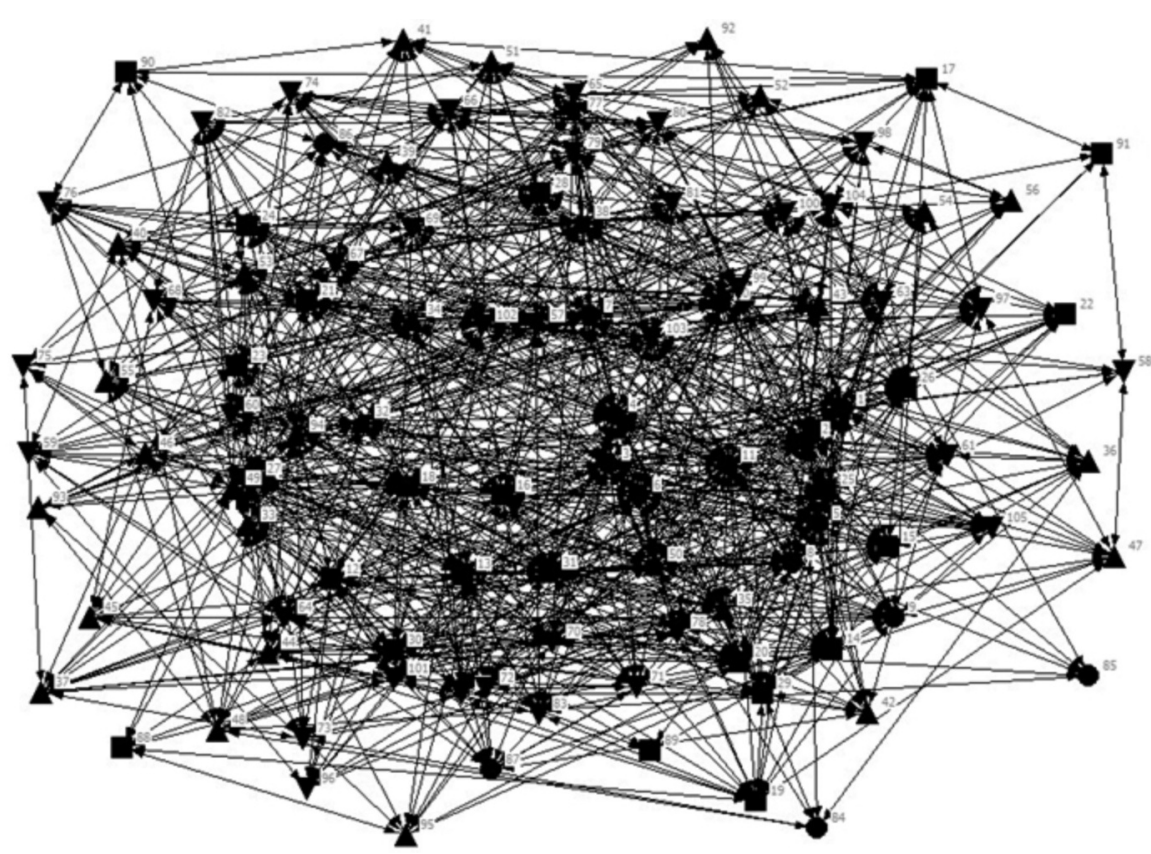

Leyenda de los principales agentes

1 Oficina de Turismo Figueres

2 Oficina de Turismo de Rosas

3 Oficina de Turismo de Portbou

4 Oficina de Turismo de Castelló d'Empúries

5 Oficina de Turismo de Peralada

6 Oficina de Turismo de Sant Pere Pescador

11 Oficina de Turismo de Llançà

33 Consejo Regulador del Vino del Empordà

40 Ayuntamiento de Roses

41 Ayuntamiento de Figueres

43 Ayuntamiento de Peralada

45 Ayuntamiento de Garriguella

46 Ayuntamiento de Sant Climent Sescebes

49 Alt Empordà Turisme

50 Ayuntamiento de Villajuiga

52 Ayuntamiento de Campmany

57 Consorcio del Castell de Peralada

67 Dpto de Agricultura de la Generalitat de Catalunya
68 Dirección General de Turismo de la Genaralitat de Catalunya

70 Bodega Mas Llunes

72 Celler Empordàlia

73 La Vinyeta

75 Celler Oliver Conti

77 Terra Remota

78 Cooperativa Garriguella

80 Celler Arche Pages

87 Clos d'Agon

89 Gelamà

90 Celler Cooperatiu Espolla

92 Celler Can Sais

93 Celler Marti Fabra

94 Celler Martín Faixó

95 Mas Oller

96 Masía Serra

99 Vinyes d'Olivardots

102 Vinyes del Aspres

Fuente: elaboración propia con Ucinet 6 y NetDraw. 
FIGURA 3

\section{SOCIOGRAMA RUTA 2}

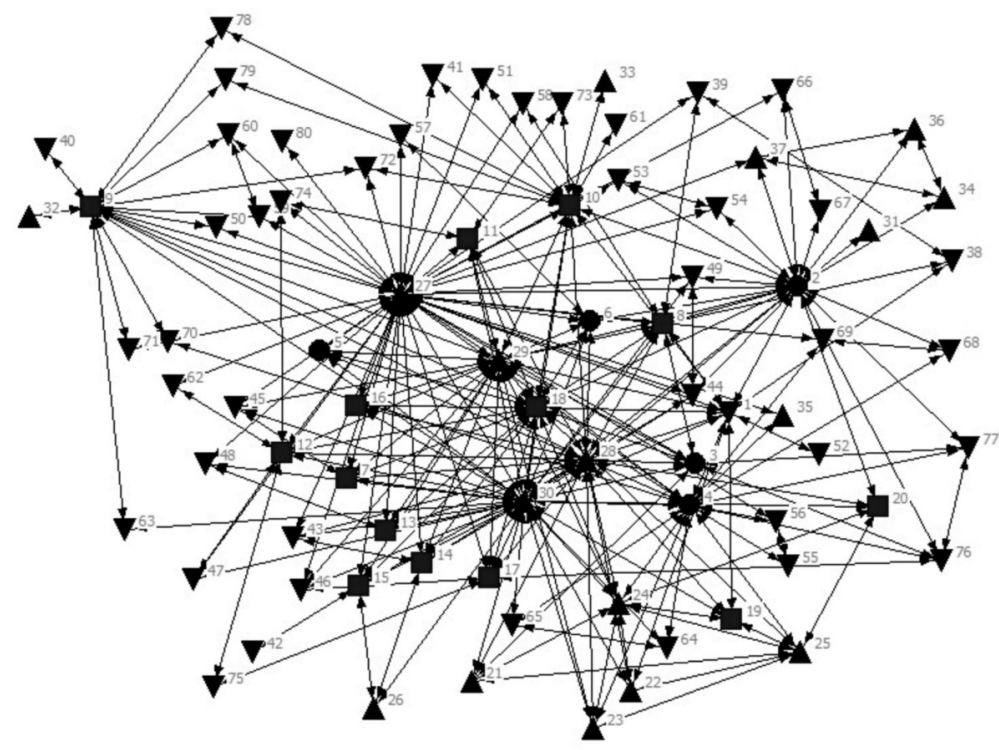

Leyenda de los principales agentes

1 Oficina de Turismo de Cornudella

2 Oficina de Turismo del Priorat

3 Oficina de Turismo de Escala Dei

8 Ayuntamiento de Cornudella

9 Ayuntamiento de Falset

10 Consell Comarcal del Priorat

11 Ayuntamiento de Ulldemolins

12 Ayuntamiento de la Morera de Montsant

15 Ayuntamiento de Gratallops

16 Ayuntamiento de Porrera

17 Ayuntamiento de Siurana

18 Ayuntamiento de Bellmunt

19 Dpto de Agricultura de la Generalitat de Catalunya

20 Dirección General de Turismo de la Genaralitat de Catalunya

27 Priorat Turisme

28 Consorci per al Desenvolupament del Priorat

29 Asociación de Cellers del Priorat
30 Asociación de la Ruta de las Bodegas

32 Costa Daurada

38 Costers del Siurana

39 Agrícola Falset-Marçà

42 Cellers Capafons-Ossó

44 Vins i Olis del Priorat

46 Vinatería Vinum

47 Viñas del Montsant

52 Celler Cal Pla

56 La Vinícola del Priorat

58 Celler Masroig

63 Cellar Mas Blanc

67 Bodegas JM Bach

68 Viticultors del Priorat

72 Marco Abella

73 Celler Els Cups

75 Celler Mas Igneus

77 Torres-Priorat

79 Celler Buil y Giné

Fuente: elaboración propia con Ucinet 6 y NetDraw. 
$\left(n^{\circ} 57\right)$. Para la ruta 2 , además de la asociación Priorat Turisme (nodo ${ }^{\circ} 27$ ), la Asociación de la Ruta de las Bodegas ( $\mathrm{n}^{\circ} 30$ ) y la Oficina de Turismo del Priorat $\left(\mathrm{n}^{\circ} 2\right)$, otros agentes con mayor grado de centralización son el Consorci per al Desenvolupament del Priorat $\left(\mathrm{n}^{\circ} 28\right)$ y la Asociación de Cellers del Priorat ( $\left.{ }^{\circ} 29\right)$.

Así pues, las oficinas de turismo son, mayoritariamente, los agentes con mayor poder de centralización en la red creada en la ruta 1, mientras que algunas de las asociaciones relacionadas con este tipo de turismo y con el desarrollo local lo hacen en la de la ruta 2.

Respecto al grado de cercanía, en la ruta 1 predominan las oficinas de turismo de Figueres (nodo ${ }^{\circ} 1$ ) y de Castelló d'Empúries ( $n^{\circ} 4$ ), el Consejo Regulador del Vino del Empordà ( $\left.n^{\circ} 33\right)$ y el Consorcio del Castell de Peralada $\left(n^{\circ} 57\right)$. Se trata de unos actores que presentan ciertas particularidades. Así, ambas oficinas de turismo están situadas en lugares estratégicos, la primera en Figures, la capital de la comarca del Alt Empordà, y la segunda en una muy poblada zona del litoral (Ampuriabrava). Por su parte, el Consejo Regulador del Vino es una organización que aglutina a los principales bodegueros de la región, ocupando los Vinos de Peralada una posición predominante, ya que, además de sus bodegas -las más importantes de la DO Empordà-, presenta una amalgama de ofertas turísticas de interés, como son el casino, el festival de música de verano, el castillo y la propia población.

En cuanto a la red de la ruta 2, los agentes con mayor grado de cercanía son Priorat Turisme (nodo $\mathrm{n}^{\circ}$ 27), la Asociación de la Ruta de las Bodegas $\left(n^{\circ} 30\right)$ y el Consorci per al Desenvolupament del Priorat ( $\left.n^{\circ} 28\right)$. Sin embargo, otros con un nivel de cercanía menor, como las Oficinas de Turismo de Cornudella y Escala Dei, por el solo hecho de estar conectadas directamente con Priorat Turismo y con la Oficina de Turismo del Priorat -agentes con más contactos-, también tienen una cierta influencia en la promoción y el desarrollo de esta ruta enológica.

En ambas redes es de destacar el papel de intermediación de los agentes públicos, tanto los Ayuntamientos como los respectivos Consells Comarcals y la propia Generalitat de Catalunya a través del departamento de Agricultura y de la Dirección General de Turismo del departamento de Empresa y Ocupación. En este aspecto, en la red del Priorat destaca el papel predominante del Ayuntamiento de Falset y del Consell Comarcal del Priorat, entidades ambas que apuestan por el turismo enológico como un medio para incentivar un desarrollo económico de la comarca basado en la industria vitivinícola.

En cuanto al ACRS, en los dos casos analizados se confirma que las relaciones establecidas en las redes generadas por los agentes involucrados con este 
tipo de turismo son multilaterales, bastante estables -casi no hay abandonos entre los miembros de las red-y suelen estar basadas en la confianza entre sus miembros para lograr un objetivo común, predominando la promoción del turismo enológico.

De esta manera, ambos territorios, que a pesar de su diferente nivel de desarrollo turístico (cuadro 1) presentan un similar desarrollo del turismo de las rutas del vino, han generado unas redes sociales alrededor de los agentes involucrados con este turismo enológico cuya dinámica relacional es similar. Sin embargo, los objetivos de ambas son distintos. Así, en las comarcas ampurdanesas, cuya estructura turística está muy consolidada, se trata de un producto que ayuda a satisfacer una demanda complementaria de los turistas, mientras que en el Priorat, casi sin estructura turística, se ha apostado claramente por el desarrollo del turismo enológico y de la industria vitivinícola, como formas de desarrollo local.

\section{CONCLUSIONES}

Como se ha podido comprobar en este trabajo con los dos casos analizados, para un destino turístico maduro y consolidado, como puede ser el Empordà, el turismo enológico -a través de las rutas del vino- es un producto innovador que, además de ayudar al desarrollo de la industria vitivinícola, es una oferta complementaria al modelo turístico tradicional, sirviéndole de valor diferencial frente a otros destinos turísticos similares y ayudando a desestacionalizar la demanda.

Por su parte, en el caso de un destino turístico poco desarrollado, como puede ser el Priorat, la puesta en valor turístico de sinergias comunes entre sus recursos naturales -en este caso, el vino, a través de los productores de la comarca- y las instituciones públicas y sus organismos asociados, además de proporcionar una oferta que cubre las nuevas demandas de los turistas, ayuda a consolidar unas marcas y una industria, lo que sirve para mejorar el desarrollo económico de unos territorios deprimidos demográfica y económicamente.

Según se ha podido observar en este trabajo, es destacable la gran implicación de las bodegas como agentes involucrados en el desarrollo de este tipo de rutas, lo cual es comprensible ya que se trata del sector empresarial más beneficiado por el crecimiento del turismo enológico. Por ello, además de las degustaciones, han ido incorporando nuevas ofertas y actividades turísticas, lo que, en muchas ocasiones, les ha supuesto realizar inversiones para mejorar 
sus instalaciones, adaptar las nuevas actividades, disponer de adecuadas infraestructuras e instalar la necesaria señalización.

También es relevante el papel que las instituciones públicas y las oficinas de turismo tienen en el desarrollo de este tipo de turismo, ejerciendo un importante papel de intermediación en las relaciones entre los agentes involucrados en estas redes de agentes, que cada vez cobran mayor importancia gracias al masivo uso de Internet como medio para obtener información acerca de los nuevos productos turísticos, sus ofertas y los destinos.

Con ello se confirma que el desarrollo de cualquier nuevo producto turístico -como son las rutas del vino y el turismo enológico-, necesita la existencia de una eficaz cooperación entre las diferentes Administraciones (central, autonómica, provincial, comarcal y local), sus organismos asociados y el sector privado (productores y comercios), lo que permite asegurar la coherencia necesaria para desarrollar con éxito cualquier proyecto de este tipo. De este modo se crean unas redes con unas relaciones de cooperación entre sus miembros que son estables, facilitando la coherencia necesaria para desarrollar el producto turístico de una forma más rápida y eficiente, tal como está sucediendo en el caso del Priorat.

Dichas relaciones reticulares juegan un papel fundamental en el desarrollo de cualquier tipo de turismo -en el caso aquí expuesto, el turismo enológico-, ya que, siguiendo a Merinero y Pulido (2009), una dinámica relacional más intensa ayuda al desarrollo del turismo. Sin embargo, tal como en este trabajo se ha podido comprobar, esta dinámica no depende del nivel general de desarrollo turístico del destino sino de la propia estructura de la red creada.

Por ello, para que un nuevo producto turístico se desarrolle eficaz y eficientemente en un territorio, sea cual sea su estructura turística, desde la oferta es necesario poner en marcha unas estrategias que ayuden, en primer lugar, a crear unas redes sociales entre los agentes involucrados con este turismo, y, posteriormente, aumentar la dinámica relacional de dichas redes, con la participación activa y comprometida de todos los agentes involucrados en el proceso, sean públicos o privados, creando unas relaciones densas, permanentes y eficaces.

Fecha de recepción: 13 de noviembre de 2012.

Fecha de aceptación: 30 de mayo de 2013. 
BIBLIOGRAFÍA

Acercosa, M. A. (2006): Marketing de destinos turísticos. Madrid, MAD.

Alonso, J. L. (2003): "Redes y procesos de innovación en las comarcas vinícolas de Castilla y León: el ejemplo de la D.O. Bierzo". BAGE, 36, pp. 43-60.

Alonso, J. L. y Méndez, R. (coord.) (2000): Innovación, pequeña empresa y desarrollo local en España. Madrid, Editorial Civitas.

Bigné, E.; Font, X. y Andreu, L. (2000): El marketing de los destinos turísticos. Madrid, ESIC.

Boniface, P. (2003): Tasting Tourism: Travelling for Food and Drink. Aldershot, Ashgate Publishing Ltd.

Borgatti, F. F; Everett, M. G. y Freeman, C. (2002): Ucinet for Windows: Software for Social Networks Analysis. Harvard, Harvard University Press.

Butler, R. (1980): "The concept of a tourism area cycle of evolution: implications for the management of resources". Canadian Geographer, 24, pp. 5-12.

Castillo, M. (2007): "Enoturismo: mucho más que una moda". Revista Alimarket, 207, pp. 59-65.

Chulwon, K. y Taesuk, L. (2005): "Exploring Four Dimensional Sources of Destination Competitiveness". International Journal of Tourism Sciences, 5/1, pp. 105-130.

Ejarque, J. (2005): Destinos turísticos de éxito: diseño, creación, gestión y marketing. Madrid, Pirámide.

Elías, L. V. (2006): El turismo del vino. Otra experiencia de ocio. Bilbao, Editorial Deusto.

Fernández Portela, J. (2012): "Cambios en la industria y en el paisaje vitivinícola de la Denominación de Origen de Cigales (Castilla y León, España)". Estudios Geográficos, LXXIII/272, pp. 63-90.

Frank, O. y Snidjers, T. (1994): "Estimating the Size of Hidden Populations Using Snowball Sampling". Journal of Official Statistics, 10/1, pp. 53-67.

Granovetter, M. (1976): "Network Sampling: some first steps". American Journal of Sociology, 83, pp. 727-729.

Hashimoto, A. y Telfer, D. J. (2006): "Selling Canadian Culinary Tourism: Branding the Global and the Regional Product". Tourism Geographies, 8/1, pp. 31-55.

Hanneman, R. A. (2000): Introducción a los métodos del análisis de redes sociales. Riverside, California University.

Haythornthwaite, C. (1996): "Social network analysis: An approach and technique for the study of information exchange". Library and Information Science Research, 18, pp. 323-342.

Hjalager, A. y Richards, G. (2002): Tourism and Gastronomy. London, Routledge.

Institut d'Estadística de Catalunya (Idescat) (2012): Estadístiques territorials. Disponible en: http: //www.idescat.cat (Fecha de consulta: 10 de Octubre de 2014).

Institut Català del Vi (Incavi) (2012): Estadístiques d'enoturisme. Barcelona, INCAVI.

International Culinary Tourism Association (ICTA) (2012): Statistics. Portland, ICTA Press. 
Johns, N. y Clarke, V. (2001): "Mythology analysis of boating tourism". Annals of Tourism Research, 28/2, pp. 334-359.

Kivela, J. y Crotts, J. C. (2006): "Tourism and gastronomy: gastronomy's influence on how tourists experience a destination". Journal of Hospitality \& Tourism Research, 30/3, pp. 354-377.

Kwangmin, P. y Jinhoo, K. (2010): "The Firm Growth Pattern in the Restaurant industry. Does Gibrats Law Hold?". International Journal of Tourism Sciences, 10/3, pp. 49-63.

Long, L. M. (ed.) (2004): Culinary tourism. Lexington, University Press of Kentucky.

Martínez Puche, A. y Jover, M. J. (coord.) (2012): "Cine y enoturismo: Representación, identidad y promoción de los territorios del vino", en A. Martínez Puche (ed.): Territorios de cine. Desarrollo local, tipologías turísticas y promoción. Alicante, Publicaciones de la Universidad de Alicante, pp. 199-220.

López-Guzmán, T. y Sánchez, S. M. (2008): "La creación de productos turísticos utilizando rutas enológicas". Pasos. Revista de Turismo y Patrimonio Cultural, 6/2, pp. 159-171.

McBee, S. (2002): To Lead Is To Serve: How To Attract Volunteers E Keep Them. South Fallsburg, NY, Shar McBee.

McCurley, S. y Lynch, R. (1996): Volunteer Management: Mobilizing All the Resources of the Community. Downers Grove, IL, Heritage Arts Publishing.

Merinero, R. y Pulido, J. I. (2009): "Desarrollo Turístico y dinámica relacional. Metodología de análisis para la gestión activa de destinos turísticos". Cuadernos de Turismo, 23, pp. 173-193.

Middleton, V. T. C. (1994): Marketing in Travel and Tourism. Oxford, ButterworthHeinemann.

Mkono, M. (2011): "The Offering of Food in Touristic Eatertainement: A Netnography". Tourist Studies, 11/3, pp. 253-270.

Molina, J. L. (2001): El análisis de redes sociales. Una introducción. Barcelona, Ediciones Bellaterra.

Mondéjar, J. A. y Sevilla, C. (2008): "Enoturismo: un producto emergente con gran potencial de crecimiento en España". Cim.economía, 13, pp. 305-327.

Observatorio Turístico Rutas del Vino de España (2010): Estudio de la Oferta Enoturística de las Rutas del Vino de España - $2^{\circ}$ semestre 2009. Madrid, Turespaña y Acevin.

Requena, F. (2003): Análisis de redes sociales. Orígenes, teorías y aplicaciones. Madrid, Centro de Investigaciones Sociológicas/Siglo XXI.

Richards, G. (2002): "Gastronomy: An essential ingredient in tourism production and consumption?", en A. M. Hjalager y G. Richards, G. (eds.): Tourism and gastro nomy. London, Routledge, pp. 3-20.

Ritchie, B. y Zins, M. (1978): "Culture as determinant of the attractiveness of a tourism region". Annals of Tourism Research, 5, pp. 226-237.

Scott, N.; Baggio, R. y Cooper, C. (2008): Network Analysis ant Tourism. From Theory to Practice. Tonawanda, NY, Channel View Publications. 
Smith, S. y Xiao, H. (2008): "Culinary Tourism Supply Chains: A Preliminary Examination". Journal of Travel Research, 46/3, pp. 289-299.

Vogeler, C. y Hernández, E. (2000): El mercado turístico: estructura, operaciones y procesos de producción. Madrid, Centro de Estudios Ramón Areces.

Warde, A. (1997): Consumption, Food and Taste. London, Sage.

Wolf, E. (2002): Culinary tourism: A tasty economic proposition. Portland, Culinary Tourism Taskforce,

Zillinger, M. (2007): "Tourist routes: A Time-Geographical Approach on German CarTourists in Sweden”. Tourism Geographies, 9/1, pp. 64-83.

\section{RESUMEN}

En los destinos turísticos, las nuevas necesidades y motivaciones de los turistas, cada vez más experimentados, que demandan una cierta personalización de los productos turísticos, el desarrollo de nuevos productos es una de las estrategias más generalizadas. Uno de ellos, el turismo enológico ha desarrollado las rutas del vino para satisfacer estas demandas. Por ello, con el objetivo de comprobar si la dinámica creada por las relaciones generadas en las redes sociales de los agentes involucrados con este turismo (productores, asociaciones, oficinas de turismo e instituciones públicas) tiene una relación directa con el grado de desarrollo del mismo, en el presente trabajo se ha realizado el análisis de estas redes sociales y de sus contenidos en el Empordà y el Priorat, comparándose sus resultados y confirmándose que existe una relación directa entre ambos factores -desarrollo y dinámica relacional- independientemente del nivel de desarrollo del turismo en el destino.

Palabras Clave: turismo; rutas turísticas; enoturismo; relaciones sociales; análisis de redes sociales; desarrollo local.

\section{Abstract}

In the tourist destinations, the new needs and motivations of the tourists, increasingly experienced, that ask a certain customization of the touristic products, the development of new products is one of the strategies most generalized. One of them, the enologic tourism has development the routes of wine in order to satisfier these demands. By it, with the aim to verify if the dynamics created about the relations generated in the social networks of the agents involved with the development of this tourism (producers, associations, offices of tourism and public institutions) has a direct relation with the degree of development of the same one, in the present work there has been realized the analysis of these social networks and of his contents in the the Empordà and the Priorat, his results being compared and being confirmed that exists a direct relation between both factors -development and relational dynamics- with independence del level of development of the tourism in the destination.

KEY WORDS: tourism; tourist routes; enotourism; social relations; analysis of social networks; local development. 


\section{RÉSUMÉ}

Dans les destinations touristiques, les nouvelles nécessités et les motivations des touristes, de plus en plus expérimentés, qu'ils demandent certaine personnalisation des produits touristiques, le développement de nouveaux produits est l'une des stratégies les plus généralisées. Ainsi, les routes du vin se présentent comme une activité du tourisme enologique qui satisfait ces demandes. Par cela, avec l'objectif de vérifier si la dynamique créée autour des relations générées dans les réseaux sociaux des agents impliqués avec le développement de ce type de tourisme (producteurs, associations, bureaux de tourisme et institutions publiques) a une relation directe avec le degré de développement de même, au travail présent on a réalisé l'analyse de ces réseaux sociaux et de ses contenus dans l'Empordà et le Priorat, ses résultats étant comparés et en se confirmant que il y a une relation directe entre les deux facteurs -développement et dynamique relational- avec indépendance du niveaux du développement du tourisme dans la destination.

Mots CLES: tourisme; routes touristiques; enotourisme; relations sociales; analyse de réseaux sociaux; développement local. 